LIVER

\title{
Bile secretory function in the obese Zucker rat: evidence of cholestasis and altered canalicular transport function
}

\author{
M Pizarro, N Balasubramaniyan, N Solís, A Solar, I Duarte, J F Miquel, F J Suchy, M Trauner, \\ L Accatino, M Ananthanarayanan, M Arrese
}

Gut 2004;53:1837-1843. doi: 10.1136/gut.2003.037689

See end of article for authors' affiliations

.....................

Correspondence to: Dr M Arrese, Departmento de Gastroenterología Pontificia Universidad Católica de Chile, Marcoleta \#367

Santiago 833-0024, Chile; marrese@med.puc.cl

Revised version received 25 May 2004

Accepted for publication 22 June 2004
Background: Obese Zucker rats (ZR) have been used as an experimental model for non-alcoholic fatty liver disease and are particularly susceptible to various types of liver injury. Bile secretory function has not been assessed in ZR.

Aim: To study bile secretion and expression of the main hepatobiliary transporters in ZR.

Methods: Bile flow and biliary secretion of lipids and glutathione were determined in eight and 14 week old obese ZR and their lean controls. Protein mass and mRNA of the $\mathrm{Na}^{+} /$taurocholate cotransporting polypeptide (Ntcp), the bile salt export pump (Bsep), and the multidrug resistant associated protein 2 (Mrp2) were assessed by western and northern blot, respectively. The effects of administration of a tumour necrosis factor $\alpha$ inactivator (etanercept) and an insulin sensitiser (rosiglitazone) were assessed in obese ZR while leptin was given to non-obese rats to study its effect on Mrp2 expression.

Results: ZR exhibited increased body weight and hyperlipidaemia. Only 14 week old obese ZR has fatty liver. Decreased bile flow and biliary lipid and glutathione secretion as well as reduced hepatic transport of both taurocholate and bromosulphthalein were found in obese ZR. Hepatic Mrp2 protein mass was markedly reduced $(-70 \%)$ in obese rats while Ntcp and Bsep protein levels were similar to lean rats. Downregulation of Mrp2 seems to involve both transcriptional and post-transcriptional mechanisms probably related to insulin and leptin resistance.

Conclusions: Obese ZR exhibit an impaired bile secretory function with significant functional and molecular alterations consistent with mild cholestasis. A defective hepatobiliary transport capacity may be a contributory factor in rendering the obese ZR more susceptible to liver injury.
$\mathrm{N}$ on-alcoholic fatty liver disease (NAFLD) is an increasingly recognised clinicopathological condition that comprises a wide spectrum of liver damage ranging from simple steatosis to steatohepatitis, advanced fibrosis, and cirrhosis. ${ }^{1-3}$ The underlying mechanisms involved in the transition from uncomplicated steatosis to more advanced stages of the disease are not fully understood..$^{4-6}$ Data gathered from both humans and experimental animals suggest that fatty livers are more prone to a variety of insults such as endotoxin ${ }^{7}$ and ischaemia/reperfusion (I/RP). ${ }^{9}$ The observed increased susceptibility to injury gains importance when an hepatic injury occurs in a fatty liver as it may be followed by an exaggerated hepatocyte necrosis and apoptosis and subsequently by an inflammatory process which in turn may trigger a fibrogenic response resulting in significant collagen deposition. ${ }^{6}$

Animal studies showing increased susceptibility or decreased tolerance of steatotic livers to injury have been conducted mainly in genetically obese rodents such as the ob/ob mice or the obese Zucker rat (ZR). These rodents have defective brain leptin dependent signal transduction due to either lack of leptin production or a dysfunctional leptin receptor, resulting in markedly increased food intake and decreased energy expenditure, which are associated with obesity, insulin resistance, and fatty liver. ${ }^{10}$ Both ob/ob mice and obese ZR displayed increased hepatotoxicity and decreased survival after exposure to endotoxin and had increased hepatic injury and decreased survival after 60 minutes of ischaemia compared with lean littermates. ${ }^{71}$ However, the specific underlying mechanisms explaining the observed increased sensitivity to liver injury observed in these experimental models of NAFLD are not well known. ${ }^{12}{ }^{13}$
Several studies have provided evidence of altered Kupffer cell function and increased hepatocyte sensitivity to tumour necrosis factor $\alpha$ (TNF- $\alpha$ ) as relevant phenomena in obesity related liver sensitivity to endotoxin. ${ }^{73}{ }^{14}$ Additionally, increased lipid peroxidation, neutrophil infiltration, and increased release of TNF- $\alpha$ as well as microcirculatory alterations may also be responsible for the higher rate of dysfunction after I/RP in fatty livers. ${ }^{15}$

Bile secretion from the liver has a pivotal physiological role as an excretory route for endo- and xenobiotics. ${ }^{16}$ During bile secretory failure or cholestasis, substances normally secreted into bile accumulate inside the cell promoting liver injury and leading to both necrosis and apoptosis. In recent years, the molecular mechanisms of bile formation as well the adaptive changes occurring during cholestasis have been well characterised. ${ }^{17}{ }^{18}$ The concept that malfunction of hepatobiliary transport proteins is associated with cholestasis and liver injury is now established due to identification of a number of inherited diseases, which are caused by mutations in hepatic solute pumps. ${ }^{19}$ As bile secretory function has not been studied in detail in genetically obese animals and disturbed bile formation may be a contributory factor in rendering the fatty liver more susceptible to injury, the present study was

Abbreviations: NAFLD, non-alcoholic fatty liver disease; I/RP, ischaemia/reperfusion; ZR, Zucker rat; TNF- $\alpha$, tumour necrosis factor $\alpha$; BS, bile salts; AST, aspartate aminotransferase; ALT, alanine aminotransferase; Ntcp, $\mathrm{Na}^{+}$/taurocholate cotransporting polypeptide; Bsep, bile salt export pump; Mrp, multidrug resistant associated protein; GAPDH, glyceraldehyde-3-phosphate dehydrogenase; TC, taurocholate; BSP, bromosulphthalein; SRm, maximum secretory rate; PPAR- $\gamma$, proliferator activated receptor $\gamma$; RGZ, rosiglitazone; PCR, polymerase chain reaction 
carried out to assess bile secretory function in the obese ZR as well as expression of major hepatobiliary transporter proteins of the liver in these animals. Our results show that the obese ZR have significant functional alterations of bile secretory function, consistent with the presence of cholestasis, and exhibit decreased expression and/or function of some critical canalicular transporters resulting in impaired excretory function of the liver.

\section{EXPERIMENTAL PROCEDURES}

\section{Animals}

Male lean (?/fa) and obese (fa/fa) ZR (Charles River Laboratories, Wilmington, Massachusetts, USA) of eight and 14 weeks of age were housed in transparent polycarbonate cages and subjected to 12 hour light/darkness cycles at a temperature of $21^{\circ} \mathrm{C}$ and a relative humidity of $50 \%$ throughout the accommodation (at least one week) period. Unless otherwise stated, all animals had free access to standard rodent chow and tap water. Animal experiments were approved by the local ethics review committee on animal experiments according to the criteria outlined in the Guide for the Care and Use of Laboratory Animals prepared by the National Academy of Sciences and published by the National Institutes of Health (NIH publication 86-23, revised 1985). Animals were anaesthetised with a single dose of sodium pentobarbital ( $50 \mathrm{mg} / \mathrm{kg}$ body weight intraperitoneally) and bile collection was carried out after cannulation of the proximal common bile duct with a PE-10 polyethylene tube just proximal to the bifurcation. Subsequently, livers were removed after a short perfusion with saline solution.

\section{Analytical procedures}

Bile flow was measured gravimetrically and total biliary bile salts (BS) were quantitated by the 3-alpha-hydroxysteroiddehydrogenase method. ${ }^{20}{ }^{21}$ Total biliary phospholipids and biliary cholesterol were measured by standard methods. ${ }^{22}{ }^{23}$ Serum aspartate aminotransferase (AST), alanine aminotransferase (ALT), and total BS were measured using a Merck Diagnostic Kit (E Merck, Darmstadt, Germany). Serum triglycerides, glucose, and cholesterol were measured using a kit from Human Gesselheit (Wiesbaden, Germany). Serum alkaline phosphatase was measured by conventional techniques. Glutathione in liver and bile was determined as described by Anderson ${ }^{24}$ and $\gamma$-glutamylcysteine synthetase, the rate limiting enzyme in glutathione synthesis, was determined spectrophotometrically according to Seeling and Meister. ${ }^{25}$ Conventional histological studies were performed using the haematoxylin-eosin stain.

\section{Expression of hepatic transporter proteins}

Protein mass of hepatic transporters in liver tissue from lean and obese rats was measured as previously described ${ }^{26}$ using membrane rich fractions to assess protein expression of the main bile acid importer $\mathrm{Na}^{+}$/taurocholate cotransporting polypeptide (Ntcp, Slcl0al), the canalicular bile salt export pump (Bsep, Abcbll), and the canalicular export pump for organic anions (Mrp2, Abcc2). In addition, we measured protein levels of the basolateral Mrp homologue Mrp3 (Abcc3), which functions as an inducible export pump for bilirubin conjugates and BS. ${ }^{18}$ Western blot analysis was performed with standard techniques using the Renaissance western blotting kit (New England Nuclear, Boston, Massachusetts, USA). Membranes were probed with an anti-Ntcp fusion protein IgG, as previously described, ${ }^{27}$ a polyclonal anti-rat Bsep antibody against a C terminus 13 amino acid sequence, ${ }^{28}$ a polyclonal rabbit anti-Mrp2 raised against the $\mathrm{C}$ terminus 12 amino acid sequence, ${ }^{29}$ and a polyclonal rabbit anti-Mrp3 (kindly provided by Carol
Soroka, PhD). Immunoreactive bands were quantified by laser densitometry.

To determine Ntcp, Bsep, and Mrp2 mRNA levels, total RNA was isolated from whole liver tissue by acid guanidinium thiocyanate-phenol chloroform extraction. ${ }^{30}$ Poly(A) RNA was isolated using oligo $(\mathrm{dT})_{\mathrm{n}}$ linked to magnetic streptavidin beads with the PolyATtract mRNA Isolation System IV (Promega, Madison, Wisconsin, USA). Northern blots were performed as previously described. ${ }^{26}$ Expression levels of glyceraldehyde-3-phosphate dehydrogenase (GAPDH) were used as a control as this gene is known to be upregulated in obese ZR. ${ }^{31}{ }^{32}$ Differences in loading were corrected after reprobing the stripped blots for the "housekeeping" gene cyclophilin. The following cDNA probes were used in the northern blotting analysis: (1) Ntcp: $0.9 \mathrm{~kb}$ EcoRI fragment (Genbank M77479) isolated from the full length cDNA cloned into pBluescript; (2) Bsep: $4.0 \mathrm{~kb}$ Xho I fragment (Genebank U69487) isolated from the full length cDNA cloned into pcDNA3; (3) Mrp2: $2.5 \mathrm{~kb}$ fragment encoding the C terminal half of cDNA (Genebank L49379) amplified by polymerase chain reaction (PCR) and cloned into pCR2.1; (4) GAPDH: a $1.3 \mathrm{~kb}$ Pst I fragment isolated from full length cDNA cloned into pGEM3 (Promega Corporation); and (5) cyclophilin: a $1.3 \mathrm{~kb}$ rat cyclophilin cDNA obtained by digestion with Pst I of pGEM3 plasmid, as described previously. ${ }^{33}$ All amplified reverse transcriptionPCR products were verified by sequencing (automated fluorescent sequencing) and BLAST analysis against the Genebank database.

\section{Transport studies and tissue immunofluorescence}

The function of the canalicular transporters of both BS (Bsep) and organic anions (Mrp2), which are largely responsible for the generation of bile flow, was studied by determining the maximum secretory rate (SRm) of sodium taurocholate (TC) and bromosulphthalein (BSP) using previously described protocols. $^{34}{ }^{35}$ In addition, qualitative distribution of both Bsep and Mrp2 was assessed using indirect immunofluorescence, as described previously. ${ }^{26}$ Digital images were recorded and processed using Adobe Photoshop (Adobe Systems Inc., San Jose, California, USA).

\section{Pharmacological treatments}

Etanercept, a TNF- $\alpha$ binding moiety derived from soluble TNF- $\alpha$ receptor subunits (Embrel; Wyeth Pharmaceuticals, Madison, New Jersey, USA) was given to obese ZR to study the effects of neutralisation of this inflammatory cytokine on bile secretory function using the protocol described by Geier and colleagues. ${ }^{36}$ The effects of reduction of insulin resistance in obese animals was studied after administration of the proliferator activated receptor $\gamma$ (PPAR- $\gamma$ ) agonist rosiglitazone ( $3 \mathrm{mg} / \mathrm{kg} /$ day orally over seven days), as described previously. ${ }^{37}$

Finally, in order to assess the effects of leptin on bile secretory function and protein mass of canalicular bile salt and organic anion transporters, we carried out experiments involving induction of moderate hyperleptinaemia in 14 week old non-obese animals, as described by Barazzoni and colleagues. ${ }^{38}$ Animals underwent either leptin (Research Diagnostics Inc, New Jersey, USA) $(\mathrm{n}=3$, rate of infusion $0.4 \mathrm{mg} / \mathrm{kg} /$ day $)$ or vehicle $(\mathrm{n}=3,5 \mathrm{mmol} / \mathrm{l}$ sodium citrate, $\mathrm{pH}$ 7.4) infusion via osmotic minipumps implanted subcutaneously in the back (ALZET 2MLl; Alzet Corporation, Palo Alto, California, USA). Vehicle infusion animals were pair fed to leptin treated animals.

\section{Statistics}

All results are expressed as mean (SEM). A two tailed nonpaired Student's $t$ test was used to compare differences 
Table 1 Body weight, liver weight, and plasma parameters of lean and obese Zucker rats

\begin{tabular}{|c|c|c|c|c|}
\hline & \multicolumn{2}{|c|}{8 week old ZR } & \multicolumn{2}{|c|}{14 week old ZR } \\
\hline & Lean $(? / \mathrm{fa})$ & Obese $(\mathrm{fa} / \mathrm{fa})$ & Lean $(? / \mathrm{fa})$ & Obese $(f a / f a)$ \\
\hline Body weight (g) & $198(7.4)$ & $251.2(7.9)^{\star}$ & $263.4(21.7)$ & $411.0(17.4)^{*}$ \\
\hline Liver weight (g) & $6.2(0.21)$ & $9.4(0.36)^{*}$ & $7.1(0.4)$ & $12.8(0.9)^{*}$ \\
\hline Body/liver weight & $32.1(0.7)$ & $26.8(0.76)^{*}$ & $36.6(1.3)$ & $33.0(1.9)$ \\
\hline Glucose (mg/dl) & $132.9(6.5)$ & $151.2(9.1)^{\star}$ & $119.6(2.9)$ & $191.9(12.6)^{*}$ \\
\hline AST (U/I) & $84.8(4.3)$ & $193.4(19.4)^{*}$ & $110.5(5.8)$ & 147.7 (13.5) \\
\hline ALT (U/I) & $46.9(4.9)$ & $58.0(0.2)$ & $67.8(6.1)$ & $66.6(5.0)$ \\
\hline Cholesterol (mg/dl) & $72.1(2.6)$ & $123.9(32.2)^{*}$ & $74.1(8.0)$ & $96.0(8.8)$ \\
\hline Triglycerides (mg/dl) & $33.7(2.9)$ & $293.8(59)^{*}$ & $40.4(7.1)$ & $418.2(68.7)^{*}$ \\
\hline Bilirubin $(\mu \mathrm{mol} / \mathrm{ll})$ & $9.0(1.0)$ & $10.9(1.3)$ & $4.4(0.23)$ & $4.4(1.4)$ \\
\hline
\end{tabular}

between groups. Values were considered significantly different when the $\mathrm{p}$ value was equal to or less than 0.05 .

\section{RESULTS}

\section{Body and liver weight, serum biochemistry, and liver} histology

As expected, both eight and 14 week old male obese rats had significantly greater body weights, serum glucose concentrations, and serum lipid levels than their lean male littermates (table 1). Serum BS levels were found to be elevated in 14 week old animals (7.5 (0.96) v 15.8 (2.7) mmol/l in control and obese animals, respectively) while no differences were found in serum alkaline phosphatase levels. Total liver weights of obese rats were greater than those of lean controls. When liver weight was normalised to body weight, the ratio was reduced in eight week old obese ZR and comparable with lean rats in 14 week old obese ZR. This correlates with the absence of significant hepatic steatosis on histological examination in younger obese animals (fig 1) who do not have a fully developed metabolic syndrome ${ }^{39}$ and display mainly peripheral obesity. In the case of 14 week old obese ZR, livers exhibited significant steatosis $(>20 \%$ of hepatocytes) without significant inflammation. Finally, serum AST levels were significantly increased in obese ZR compared with their lean littermates at both age points studied while no changes were observed in serum levels of either total bilirubin or ALT.

\section{Assessment of bile secretory function}

Table 2 shows values obtained after determination of bile flow, bile salt secretion, biliary lipid (cholesterol and phospholipid), and glutathione secretion as well as hepatic glutathione content and SRm of TC and BSP in both eight week and 14 week old obese and lean ZR. An impaired bile secretory function was found in obese ZR at both time points studied, which was mainly characterised by a significant reduction in bile flow by $45-55 \%$, indicating the presence of cholestasis. ${ }^{40}$ In contrast, while biliary lipid secretion, biliary
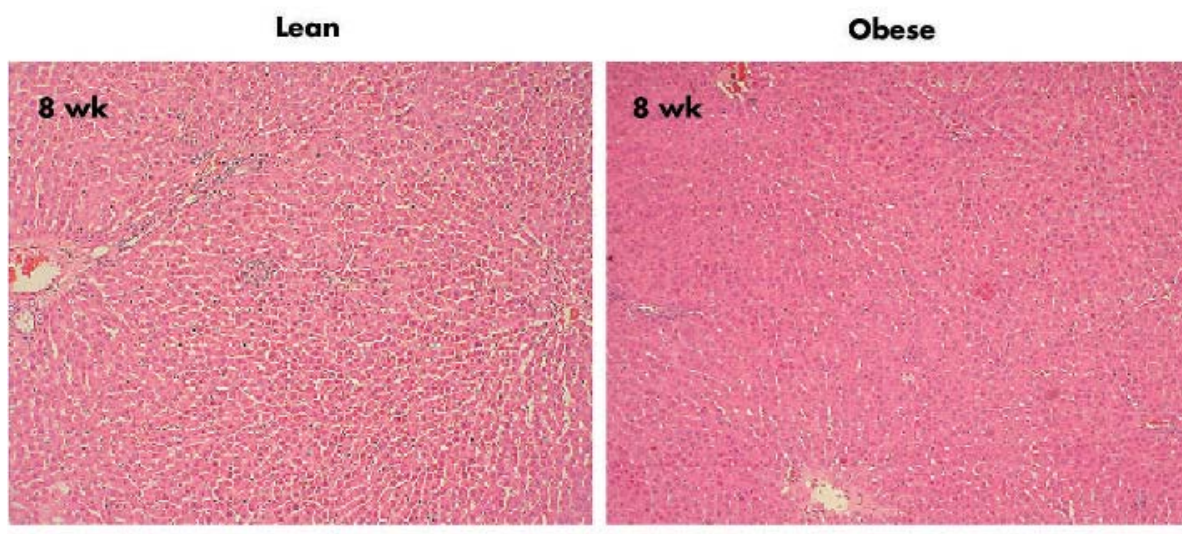

Figure 1 Liver histology in eight and 14 week old lean and obese Zucker rats. Micro- and macrovesicular steatosis was observed in a representative 14 week old obese rat while the other liver specimens displayed normal liver histology (final magnification $200 \times$ ).
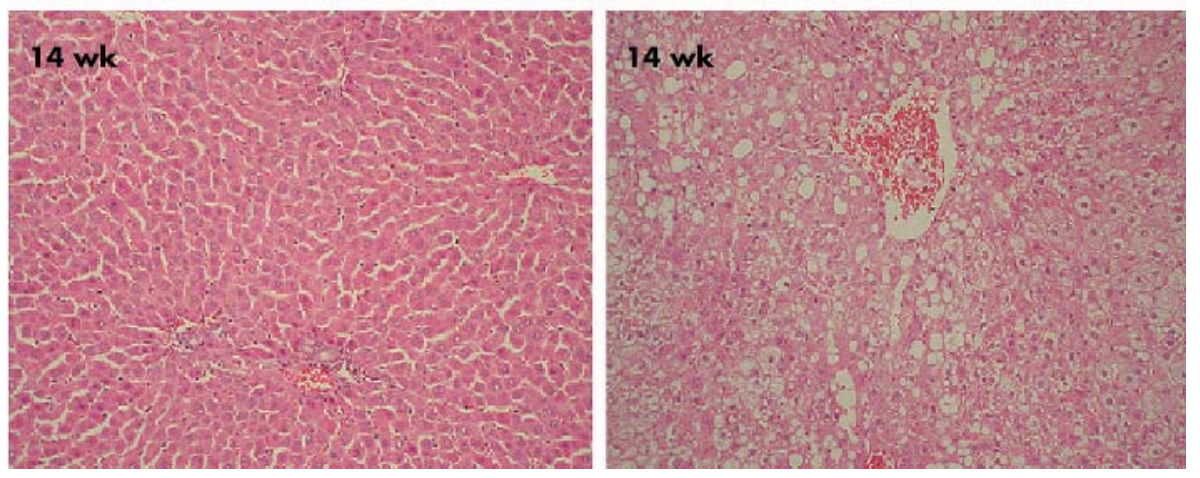
Table 2 Bile flow, biliary secretion of biliary lipids and glutathione, hepatic glutathione content, and maximal bromosulphthalein transport in lean and obese Zucker rats

\begin{tabular}{|c|c|c|c|c|}
\hline & \multicolumn{2}{|c|}{8 week old ZR } & \multicolumn{2}{|c|}{14 week old ZR } \\
\hline & Lean $(? / \mathrm{fa})$ & Obese $(\mathrm{fa} / \mathrm{fa})$ & Lean $(? / \mathrm{fa})$ & Obese $(\mathrm{fa} / \mathrm{fa})$ \\
\hline Bile flow $(\mu \mathrm{l} / \mathrm{min} \times \mathrm{g}$ liver $)$ & $2.9(0.2)$ & $1.6(0.2)^{*}$ & $3.1(0.17)$ & $1.4(0.11)^{*}$ \\
\hline Glutathione (nmol/min $\times \mathrm{g}$ liver) & $5.5(0.7)$ & $1.1(0.5)^{*}$ & $8.7(1.0)$ & $0.88(038)^{*}$ \\
\hline Bile salts $(\mathrm{nmol} / \mathrm{min} \times \mathrm{g}$ liver) & $92.2(8.5)$ & $36.9(8.8)^{\star}$ & $64.7(8.0)$ & $44.7(8.2)$ \\
\hline Cholesterol (nmol/min ×g liver) & $4.3(0.8)$ & $1.0(0.13)^{*}$ & $2.3(0.5)$ & $0.83(0.1)^{*}$ \\
\hline Phospholipids ( $\mathrm{nmol} / \mathrm{min} \times \mathrm{g}$ liver) & $4.0(0.6)$ & $1.4(0.3)^{*}$ & $3.8(0.4)$ & $1.84(0.3)^{*}$ \\
\hline Liver glutathione (nmol/mg liver) & $3.9(0.5)$ & $4.3(0.26)$ & $4.6(0.6)$ & $4.3(0.45)$ \\
\hline TC SRm (nm/min $\times g$ liver) & $390(32)$ & $274(26.9)^{*}$ & $278.6(5.5)$ & $193.8(20.4)^{*}$ \\
\hline BSP SRm $(\mu \mathrm{g} / \mathrm{min} \times \mathrm{g}$ liver) & $29.7(1.1)$ & $18.8(3.2)^{*}$ & $38.3(3.5)$ & $17.2(038)^{*}$ \\
\hline
\end{tabular}

Data represent mean (SEM), $n=4-6$ per group.

ZR, Zucker rat; SRm, maximal secretory rate; TC, taurocholate; BSP, bromosulphthalein.

${ }^{*} p<0.05$ compared with respective lean control.

glutathione secretion, and maximum transport of TC and BSP were reduced in both eight and 14 week old obese ZR, basal biliary secretion of BS was significantly reduced only in eight week old animals. In addition, hepatic glutathione content and $\gamma$-glutamylcysteine synthetase activity (data not shown) were similar in ZR and lean controls at both ages studied, suggesting that reduced biliary glutathione excretion is probably due to a defect in canalicular transport of this tripeptide.

\section{Protein expression levels of hepatobiliary transporters}

Immunoblotting was performed on liver membrane fractions from obese ZR and their lean littermates at eight and 14 weeks of age to determine if the impaired bile secretory function observed in obese rats correlated with changes in protein mass of the major hepatobiliary transporters. While no changes were observed in the protein mass of Ntcp, the main sinusoidal bile acid importer of the hepatocyte (data not shown), obese ZR had significantly reduced Mrp2 protein levels at both time points studied (fig 2). Obese ZR had an $80 \%$ and $60 \%$ reduction in Mrp2 protein mass at eight and 14 weeks old, respectively. On the other hand, Bsep protein levels were similar in lean and obese ZR. Finally, Mrp3 protein levels, which generally increase when Mrp2 levels are reduced, ${ }^{18}{ }^{41}$ remained unchanged, indicating that decreased Mrp2 expression is not necessarily accompanied by increased Mrp3 expression, as reported by Cao and colleagues. ${ }^{42}$

\section{mRNA expression levels of hepatic transporters}

Steady state mRNA levels of canalicular transporters were quantified using northern blot analysis. As shown in fig 3, a significant decrease in expression of both Mrp2 and Bsep mRNA levels was observed, being more prominent at 14 weeks of age. Mrp2 levels at eight weeks of age were not significantly decreased compared with lean controls while there was a $64 \%$ decrease at 14 weeks of age. mRNA levels for Bsep were decreased at both eight and 14 weeks of age by $46 \%$ and 53\%, respectively. Consistent with previous reports $^{33}$ we observed increased expression of GAPDH mRNA levels in obese ZR even though the difference was statistically significant only at 14 weeks of age.

\section{Bsep and Mrp2 tissue immunofluorescence}

To assess qualitative distribution of the main canalicular transporters Bsep and Mrp2, we performed indirect immunofluorescence in 14 week old obese ZR and their lean littermates. Figure 4 shows representative images of immunostaining of Mrp2, which was downregulated in obese ZR, demonstrating decreased labelling of the canalicular membrane in livers from obese rats. Bsep labelling (not shown) remained unchanged.

\section{Assessment of potential mechanisms of Mrp2 downregulation in obese Zucker rats}

In attempting to address the underlying mechanism of the observed downregulation of the xenobiotic transporter Mrp2 in ZR, we conducted experiments involving neutralisation of
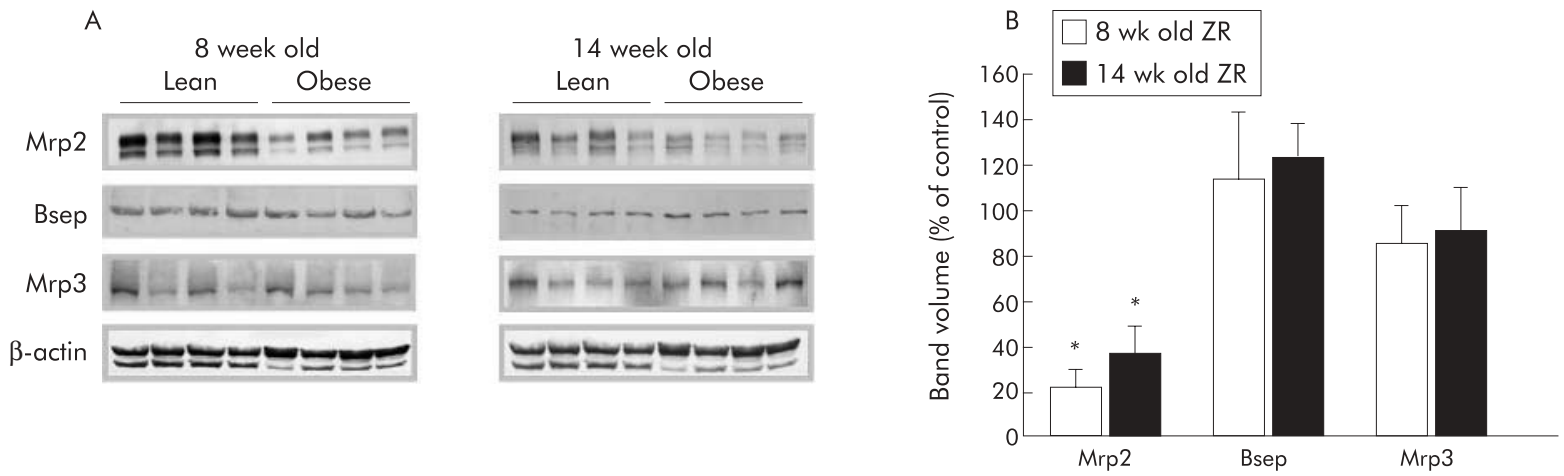

Figure 2 Protein expression of hepatic transporters in eight and 14 week old lean and obese Zucker rats (ZR). (A) Multidrug resistant associated protein 2 (Mrp2), bile salt export pump (Bsep), and multidrug resistant associated protein 3 (Mrp3) protein levels in plasma membrane fractions of lean and obese ZR livers. Approximately $50 \mu \mathrm{g}$ of membrane proteins were subjected to sodium dodecyl sulphate-polyacrylamide gel electrophoresis and transferred onto a nitrocellulose membrane. Immunoblotting was performed and bound antibodies were visualised as described in materials and methods. Each band represents the results of a single animal. Transport proteins are indicated on the left of each blot and animal groups at the top. Representative experiments with four rats per group are shown. $\beta$-Actin protein mass was used as a loading control. (B) Bar diagram showing western blot band volume as per cent of control for Mrp2, Bsep, and Mrp3. ${ }^{*} p<0.05$. 
A

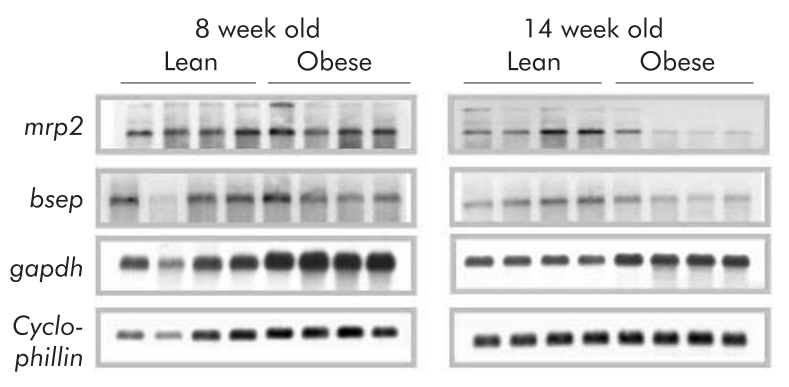

B

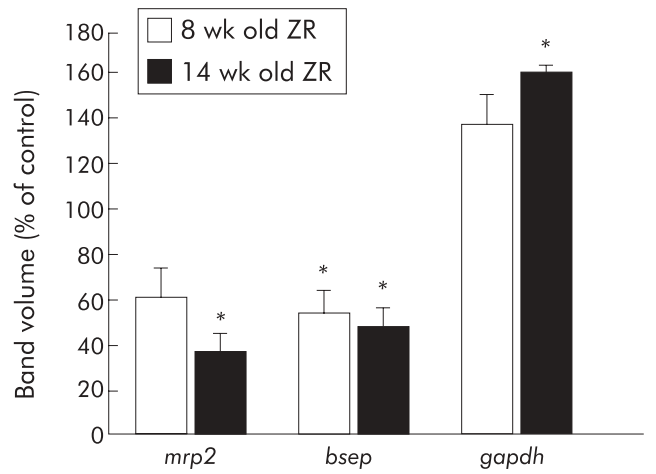

Figure 3 Decreased expression of hepatic canalicular transporter mRNA levels in eight and 14 week old Zucker rats (ZR). (A) Multidrug resistant associated protein 2 (mrp2), bile salt export pump (Bsep), and glyceraldehyde-3-phosphate dehydrogenase (gapdh) mRNA expression in lean and obese ZR. Total RNA was isolated from lean and obese animals and $5 \mu \mathrm{g}$ polyA+RNA from each sample was fractionated on $1 \%$ formaldehydeagarose probed with cDNAs for the various genes, as described in materials and methods. Each band represents the results of a single animal. Gene names are indicated on the left of each gel and animal groups at the top. (B) Bar diagram showing relative mRNA expression levels of mrp2, Bsep, and gapdh normalised to cyclophilin expression. * $\mathrm{p}<0.05$ compared with controls.

the inflammatory cytokine TNF- $\alpha$ in obese rats using the anti-TNF- $\alpha$ inhibitor etanercept, reduction of insulin resistance by treating obese ZR with the PPAR- $\gamma$ agonist rosiglitazone, and induction of hyperleptinaemia in nonobese animals. After administration of etanercept we did not found any significant changes in bile secretory function parameters (bile flow, bile salt secretion, or glutathione secretion; data not shown) or Mrp2 protein levels in etanercept treated rats compared with non-treated obese ZR (fig 5). On the other hand and in agreement with previous reports, ${ }^{37}$ treatment of obese ZR with rosiglitazone reversed some features of insulin resistance, such as hyperlipidaemia and fatty liver (data not shown), and significantly increased Mrp2 protein mass by twofold (fig 6) with only partial restorations of biliary transport abnormalities (data not shown). Finally, treatment of non-obese rats with recombinant leptin for one week induced a significant threefold increase in protein mass of Mrp2 (fig 6). Thus it seems likely that insulin and particularly leptin resistance are involved in the observed downregulation of Mrp2 in obese ZR.

\section{DISCUSSION}

The present study found evidence of mild cholestasis in obese ZR, supported by significantly reduced bile flow and elevated serum BS levels, which is consistent with this pathophysiological condition. ${ }^{40}$ Basal and stimulated BS secretion, biliary secretion of cholesterol, phospholipids, and glutathione, as well as SRm of BSP were found to be reduced in both eight and 14 week old obese ZR. Collectively, these results suggest that obese ZR have a reduced canalicular transport capacity for both BS and non-BS biliary constituents. Reduction in bile flow is mainly due to a decrease in the bile acid independent fraction and most probably related to the marked decrease in biliary glutathione secretion into bile as this tripeptide is considered the major driving force for bile acid independent flow. ${ }^{18} 4344$

Assessment of expression of several hepatic transporters responsible for the generation of bile flow, which are critical for biliary extrusion of BS and organic anions, showed that Mrp2 is markedly reduced in obese ZR. Eighty and $60 \%$ decreases in Mrp2 protein content of isolated liver plasma membrane fractions were observed in eight and 14 week old obese animals, respectively. Mrp2 downregulation appeared to involve both transcriptional and post-transcriptional events, because no changes in mrp2 mRNA levels were detected by northern blot analysis in eight week old animals but a significant decrease in Mrp2 message was seen in older animals (fig 3). Decreased Mrp2 protein levels correlated
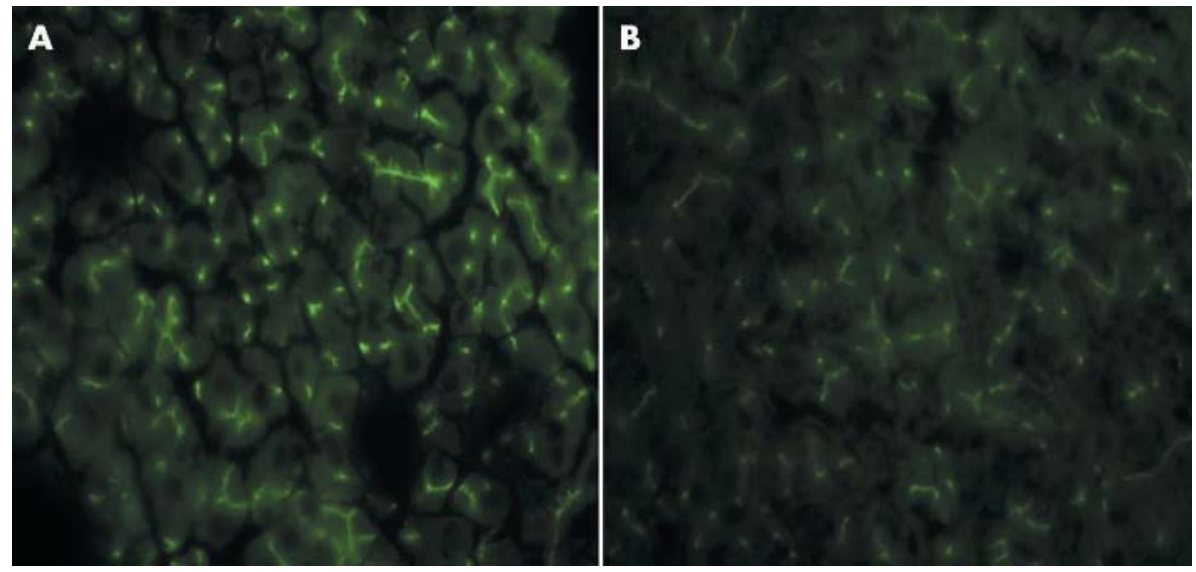

Figure 4 Indirect immunofluorescent localisation of multidrug resistant associated protein 2 (Mrp2) in 14 week old control and obese Zucker rats (ZR). Frozen liver sections from control (A) and obese (B) ZR were used to assess qualitative distribution of Mrp2 by indirect immunofluorescence, as described in material and methods Decreased labelling of canalicular membranes was observed accounting for reduction in Mrp2 protein expression in obese ZR. 

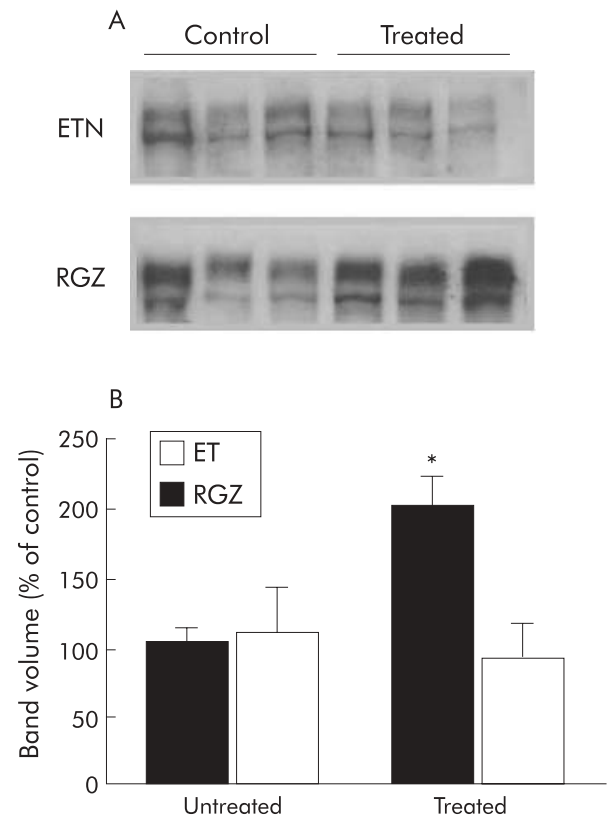

Figure 5 Effect of etanercept or rosiglitazone administration on multidrug resistant associated protein 2 (Mrp2) protein mass in obese Zucker rats (ZR). The anti-tumour necrosis factor $\alpha$ inhibitor etanercept (ETN) was given to 14 week old obese ZR in a single intraperitoneal injection of $8 \mathrm{mg} / \mathrm{kg}$. Mrp2 protein mass was assessed 72 hours later. Rosiglitazone (RGZ) was given by gavage at a dose of $3 \mathrm{mg} / \mathrm{kg} /$ day over 10 days. Mrp2 protein levels in plasma membrane fractions of treated and untreated obese rat livers were measured as described in materials and methods. (A) Each band represents the results of a single animal. Representative experiments with three rats per group are shown. (B) Bar diagram showing western blot band volume as per cent of control animals. A significant increase in Mrp2 protein levels was seen after RGZ treatment while no significant changes were observed after etanercept administration. ${ }^{*} \mathrm{p}<0.05$.

morphologically with decreased labelling in immunofluorescence studies and functionally with a decreased SRm of BSP and reduced biliary secretion of glutathione, which is mediated by Mrp2. ${ }^{45}$ Thus it is highly likely that the observed reduction in Mrp2 was responsible for the reduction in bile salt independent bile formation. Additionally, normal hepatic content and synthesis of glutathione in obese ZR (data not shown) also suggest a primary biliary transport defect of this tripeptide into bile.

In addition to Mrp2 downregulation, functional impairment of Bsep is suggested by our results. Decreased basal and maximum biliary bile salt secretion was seen in obese animals in spite of similar protein expression levels as those seen in control animals. Although the underlying mechanisms of this alteration remain to be elucidated, it is highly likely that regulatory mechanisms of this transporter are at play in obese rats $^{47}$ as mRNA levels of Bsep were reduced in both eight and 14 week old obese ZR. Further studies are required to determine factors involved in Bsep regulation in obese ZR.

The mechanisms underlying the functional and molecular alterations of bile secretion observed in the obese ZR are unclear at present. However, it can be speculated that cholestasis observed in obese ZR may result from the effects of endotoxin in the liver and the action of certain cytokines in hepatocytes. It is well known that endotoxin administration in experimental animals affects bile secretory function decreasing bile flow and bile salt secretion as well as the transport of several organic anions, including glutathione. ${ }^{48} 49$ Of note, the functional abnormalities of bile secretion induced by endotoxin are strikingly similar to those seen in
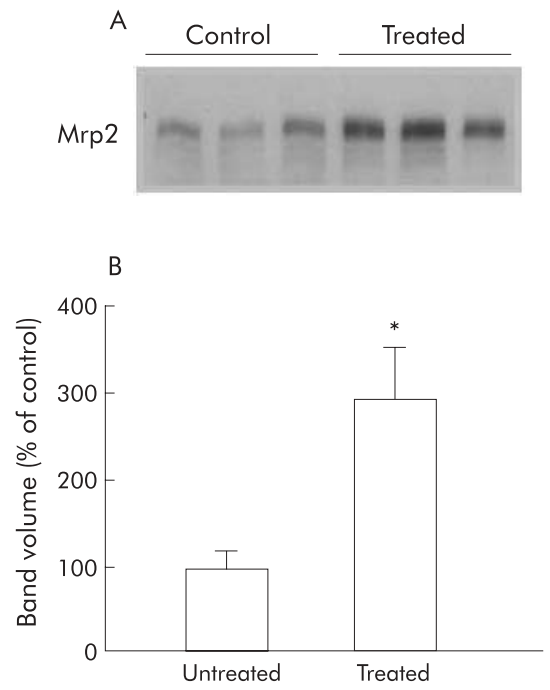

Figure 6 Effect of hyperleptinaemia on multidrug resistant associated protein 2 (Mrp2) protein mass in non-obese Zucker rats (ZR). (A) Treated rats received leptin infusion $(0.4 \mathrm{mg} / \mathrm{kg} /$ day) while control rats received vehicle $(5 \mathrm{mmol} / \mathrm{I}$ sodium citrate, $\mathrm{pH} 7.4$ ) infusion. Infusions were performed using osmotic minipumps implanted subcutaneously in the back for one week, as described in material and methods. Then, Mrp2 protein levels were measured in plasma membrane fractions of treated and untreated obese rat livers. Each band represents the results of a single animal. Representative experiments with three rats per group are shown. (B) Bar diagram showing western blot band volume as per cent of control animals. Leptin treated animals exhibited significantly higher levels of Mrp2. ${ }^{*} p<0.05$.

obese ZR. In addition, molecular assessment of hepatobiliary transporters in endotoxin treated animals have demonstrated profound downregulation of the canalicular multispecific organic anion transporter Mrp2, ${ }^{29}$ which is also present in obese ZR, a less intense decrease in Bsep, ${ }^{50}$ and a marked decrease in Ntcp. ${ }^{49}$ Both functional and molecular changes seem to be mediated by proinflammatory cytokines such as TNF- $\alpha$ and interleukin $1 \beta$. As it has been shown that obese rodents may develop intestinal bacterial overgrowth leading to modest endotoxaemia and to altered function of resident hepatic macrophages (that is, Kupffer cells) finally resulting in overproduction of cytokines, ${ }^{51}$ it is highly likely that this sequence also occurs in obese ZR. Although some differences exist between the pattern of downregulation of hepatic transporters seen in endotoxin treated animals and obese ZR (that is, obese ZR have preserved proteins levels of Ntcp which is significantly decreased after exposure to endotoxin) it is likely that those differences may be attributed to the degree of endotoxaemia seen in obese rodents. Our experiments involving neutralisation of TNF- $\alpha$ suggest that this cytokine is not the main agent responsible of Mrp2 downregulation in obese ZR. As recent reports suggest that interleukin $1 \beta$ is a more significant regulator of Mrp2 than TNF- $\alpha^{36}$ it is likely that the action of the former cytokine explains the decline in Mrp2 in obese animals.

In addition to endotoxin and cytokines, both insulin and leptin may influence the expression or activity of hepatobiliary transporters. Experiments involving reduction of insulin resistance with rosiglitazone support a role for insulin in maintaining normal levels of Mrp2, which is consistent with the reduction in Mrp2 expression seen in insulinopenic models of diabetes. ${ }^{52}$ In addition, the observation that leptin increases Mrp2 expression suggests a role for this cytokine in Mrp2 regulation. As obese ZR exhibit leptin resistance, reduced effects of leptin may account for reduced levels of Mrp2 in this rat strain. 
In summary, the present study shows that ZR exhibit impaired bile secretory function with early cholestatic changes even before the occurrence of fatty liver. Significant molecular alterations were observed in the liver of obese rats, including marked downregulation of Mrp2. These functional and molecular changes suggest the existence of a reduced hepatic ability to excrete endo- and xenobiotics in the obese ZR. We postulate that a defective hepatobiliary transport capacity may be a contributory factor in rendering ZR more susceptible to liver injury. Further studies are currently underway in our laboratory to confirm this hypothesis. If this is the case, cholestasis could be a further mechanism whereby hepatocytes laden with fat are sensitised to the development of necrosis or apoptosis when subjected to stress. ${ }^{5}$

\section{ACKNOWLEDGEMENTS}

This work was supported by grants from the Fondo Nacional de Ciencia y Tecnología a (FONDECYT \#1020641 to MA and \#1000563 to LA) and from NIH (HD20632 to FJS). Anti-rat Bsep antibody was kindly provided by Dr Bruno Stieger (Zurich, Switzerland) and antirat Mrp2 antibody was a generous gift from Dr Dietrich Keppler (Heidelberg, Germany). Anti-rat Mrp3 was provided by Dr Carol Soroka (New Haven, Connecticut, USA).

\section{Authors' affiliations}

M Pizarro, N Solís, J F Miquel, L Accatino, M Arrese, Department of

Gastroenterology, School of Medicine, Catholic University of Chile,

Santiago, Chile

N Balasubramaniyan, F J Suchy, M Ananthanarayanan, Laboratory of Developmental and Molecular Hepatology, Department of Pediatrics, Mount Sinai School of Medicine, New York, NY, USA

A Solar, I Duarte, Department of Pathology, School of Medicine, Catholic University of Chile, Santiago, Chile

M Trauner, Division of Gastroenterology, Department of Internal Medicine, University Hospital, Graz, Austria

\section{REFERENCES}

1 Alba LM, Lindor K. Non-alcoholic fatty liver disease. Aliment Pharmacol Ther 2003; 17:977-86

2 Angulo P, Lindor KD. Non-alcoholic fatty liver disease. J Gastroenterol Hepatol 2002;17(suppl):S186-90.

3 Sanyal AJ. AGA technical review on nonalcoholic fatty liver disease. Gastroenterology 2002; 123:1705-25.

4 Day CP, James OF. Steatohepatitis: a tale of two "hits"? Gastroenterology 1998; 114:842-5.

5 Day CP. Pathogenesis of steatohepatitis. Best Pract Res Clin Gastroenterol 2002; 16:663-78.

6 Friedman Sl. Liver fibrosis - from bench to bedside. J Hepatol 2003;38(suppl 1):S38-53.

7 Yang SQ, Lin HZ, Lane MD, et al. Obesity increases sensitivity to endotoxin liver injury: implications for the pathogenesis of steatohepatitis. Proc Natl Acad Sci U S A 1997;94:2557-62.

8 Haines NW, Baker AL, Boyer JL, et al. Prognostic indicators of hepatic injury following jejunoileal bypass performed for refractory obesity: a prospective study. Hepatology 1981;1:161-7.

9 Koneru B, Dikdan G. Hepatic steatosis and liver transplantation current clinical and experimental perspectives. Transplantation 2002;73:325-30.

10 Koteish A, Diehl AM. Animal models of steatosis. Semin Liver Dis 2001;21:89-104.

11 Koneru B, Reddy MC, de la Torre AN, et al. Studies of hepatic warm ischemia in the obese Zucker rat. Transplantation 1995;59:942-6.

12 Diehl AM. Nonalcoholic steatosis and steatohepatitis IV. Nonalcoholic fatty liver disease abnormalities in macrophage function and cytokines. Am J Physiol Gastrointest Liver Physiol 2002;282:G1-5.

13 Harrison SA, Diehl AM. Fat and the liver-a molecular overview. Semin Gastrointest Dis 2002;13:3-16.

14 Li Z, Lin H, Yang S, et al. Murine leptin deficiency alters Kupffer cell production of cytokines that regulate the innate immune system. Gastroenterology 2002;123:1304-10.

15 Koneru B, Dikdan G. Hepatic steatosis and liver transplantation: current clinical and experimental perspectives. Transplantation 2002;73:325-30.

16 Arrese M, Ananthananarayanan M, Suchy FJ. Hepatobiliary transport: molecular mechanisms of development and cholestasis. Pediatr Res 1998;44:141-7.

17 Arrese M, Trauner M. Molecular aspects of bile formation and cholestasis. Trends Mol Med 2003;9:558-64.

18 Trauner M, Boyer JL. Bile salt transporters: molecular characterization, function, and regulation. Physiol Rev 2003;83:633-71.
19 Elferink RO, Groen AK. Genetic defects in hepatobiliary transport. Biochim Biophys Acta 2002;1586:129-45.

20 Talalay P. Enzymatic analysis of steroid hormones. Methods Biochem Anal 1960;8:119-43.

21 Turley SA, Dietschy JM. Re-evaluation of the 3- alpha-hydroxystereiod dehydrogenase assay for total bile acids in bile. J Lipid Res 1978;19:924-8.

22 Baginski ES, Foa PP, Zak B. Microdetermination of inorganic phosphate, phospholipids and total phosphate in biological materials. Clin Chem 1967; 13:326-32.

23 Allain CC, Poon LS, Chan CS, et al. Enzymatic determination of total serum cholesterol. Clin Chem 1974;20:470-5.

24 Anderson ME. Determination of glutathione and glutathione disulfide in biological samples. Methods Enzymol 1985;113:548-55.

25 Seeling G, Meister A. Cystamine-sepharose: A probe for the active site of $\gamma$ glutamylcysteine synthetase. J Biol Chem 1982;257:5092-6.

26 Arrese M, Trauner M, Ananthanarayanan M, et al. Down-regulation of the $\mathrm{Na}^{+}$/taurocholate cotransporting polypeptide during pregnancy in the rat. $J$ Hepatol 2003;38:148-55.

27 Ananthanarayanan $\mathrm{M}, \mathrm{Ng} \mathrm{OC}$, Boyer JL, et al. Characterization of cloned rat liver $\mathrm{Na}(+)$-bile acid cotransporter using peptide and fusion protein antibodies. Am J Physiol 1994;267:G637-43

28 Gerloff T, Stieger B, Hagenbuch B, et al. The sister of P-glycoprotein represents the canalicular bile salt export pump of mammalian liver. $J$ Biol Chem 1998;273: 10046-50.

29 Trauner M, Arrese, M, Soroka CJ, et al. The rat canalicular conjugate export pump (Mrp2) is down-regulated in intrahepatic and obstructive cholestasis. Gastroenterology 1997;113:255-64.

30 Chomczynski P, Sacchi N. Single-step method for RNA isolation by acid guanidinium thiocyanate-phenol-chloroform extraction, Anal Biochem 1987; 162:156-9.

31 Rolland V, Le Liepvre X, Houbiguian ML, et al. C/EBP alpha expression in adipose tissue of genetically obese Zucker rats. Biochem Biophys Res Commun 1995;207:761-7.

32 Rolland V, Dugail I, Le Liepvre X, et al. Evidence of increased glyceraldehyde3-phosphate dehydrogenase and fatty acid synthetase promoter activities in transiently transfected adipocytes from genetically obese rats. J Biol Chem 1995;270:1102-6.

33 Hardikar W, Ananthanarayanan M, Suchy FJ. Differential ontogenic regulation of basolateral and canalicular bile acid transport proteins in rat liver. J Biol Chem 1995;270:20841-6.

34 Arrese M, Pizarro M, Solis N, et al. Adaptive regulation of hepatic bile salt transport: role of bile salt hydrophobicity and microtubule-dependent vesicular pathway. J Hepatol 1997;26:694-702.

35 Moreno M, Molina H, Amigo L, et al. Overexpression of caveolins increases bile salt secretion in mice. Hepatology 2003;38:1477-88.

36 Geier A, Dietrich CG, Voigt S, et al. Effects of proinflammatory cytokines on rat organic anion transporters during toxic liver injury and cholestasis. Hepatology 2003;38:345-54.

37 Hockings PD, Changani KK, Saeed N, et al. Rapid reversal of hepatic steatosis, and reduction of muscle triglyceride, by rosiglitazone: MRI/S studies in Zucker fatty rats. Diabetes Obes Metab 2003;5:234-43.

38 Barazzoni R, Zanetti M, Stebel $M$, et al. Hyperleptinemia prevents increased plasma ghrelin concentration during short-term moderate caloric restriction in rats. Gastroenterology 2003;124:1188-92.

39 Gabriely I, Ma XH, Yang XM, et al. Removal of visceral fat prevents insulin resistance and glucose intolerance of aging: an adipokine-mediated process? Diabetes 2002:51:2951-8.

40 Elferink RO. Cholestasis. Gut 2003;52(suppl 2):42-8.

41 Donner MG, Keppler D. Up-regulation of basolateral multidrug resistance protein 3 (Mrp3) in cholestatic rat liver. Hepatology 2001;34:351-9.

42 Cao J, Stieger B, Meier PJ, et al. Expression of rat hepatic multidrug resistance-associated proteins and organic anion transporters in pregnancy. Am J Physiol Gastrointest Liver Physiol 2002;283:G757-66.

43 Bodo A, Bakos E, Szeri F, et al. The role of multidrug transporters in drug availability, metabolism and toxicity. Toxicol Lett 2003;140:133-43.

44 Ballatori N, Truong AT. Glutathione as a primary osmotic driving force in hepatic bile formation. Am J Physiol 1992;263:G617-24.

45 Ito K, Suzuki H, Hirohashi T, et al. Functional analysis of a canalicular multispecific organic anion transporter cloned from rat liver. J Biol Chem 1998;273:1684-8

46 Wielandt AM, Vollrath V, Manzano M, et al. Induction of the multispecific organic anion transporter (cMoat/mrp2) gene and biliary glutathione secretion by the herbicide 2,4,5-trichlorophenoxyacetic acid in the mouse liver. Biochem J 1999;341:105-11.

47 Haussinger $D$, Schmitt $M$, Weiergraber $O$, et al. Short-term regulation of canalicular transport. Semin Liver Dis 2000;20:307-21.

48 Trauner M, Nathanson MH, Rydberg SA, et al. Endotoxin impairs biliary glutathione and $\mathrm{HCO} 3-$ excretion and blocks the choleretic effect of nitric oxide in rat liver. Hepatology 1997;25:1 184-91.

49 Trauner M, Arrese M, Lee $\mathrm{H}$, et al. Endotoxin downregulates rat hepatic ntcp gene expression via decreased activity of critical transcription factors. J Clin Invest 1998; 101:2092-100

50 Lee JM, Trauner M, Soroka CJ, et al. Expression of the bile salt export pump is maintained after chronic cholestasis in the rat. Gastroenterology 2000;118:163-72.

51 Li Z, Yang S, Lin H, et al. Probiotics and antibodies to TNF-alpha inhibit inflammatory activity and improve nonalcoholic fatty liver disease. Hepatology 2003;37:343-50.

52 van Waarde WM, Verkade HJ, Wolters $\mathrm{H}$, et al. Differential effects of streptozotocin-induced diabetes on expression of hepatic $A B C$-transporters in rats. Gastroenterology 2002; 122:1842-52. 